\title{
A Closed Form for Slant Submanifolds of Generalized Sasakian Space Forms
}

\author{
Pablo Alegre ${ }^{1, *}$, Joaquín Barrera ${ }^{2}$ and Alfonso Carriazo ${ }^{2,+}$ \\ 1 Departamento de Economía, Métodos Cuantitativos e Historia Económica. Área de Estadística e \\ Investigación Operativa, Universidad Pablo de Olavide. Ctra. de Utrera, km. 1. 41013 Sevilla, Spain \\ 2 Department of Geometry and Topology, Faculty of Mathematics, University of Sevilla, Apdo. Correos 1160, \\ 41080 Sevilla, Spain; barreralopezjoaquin@gmail.com (J.B.); carriazo@us.es (A.C.) \\ * Correspondence: psalerue@upo.es \\ + First and third authors are partially supported by the PAIDI group FQM-327 (Junta de Andalucía, Spain) \\ and the MEC-FEDER grant MTM2011-22621. The third author is member of IMUS (Instituto de Matemáticas \\ de laUniversidda de Sevilla).
}

Received: 4 November 2019; Accepted: 9 December 2019; Published: 13 December 2019

check for updates

\begin{abstract}
The Maslov form is a closed form for a Lagrangian submanifold of $\mathbb{C}^{m}$, and it is a conformal form if and only if $M$ satisfies the equality case of a natural inequality between the norm of the mean curvature and the scalar curvature, and it happens if and only if the second fundamental form satisfies a certain relation. In a previous paper we presented a natural inequality between the norm of the mean curvature and the scalar curvature of slant submanifolds of generalized Sasakian space forms, characterizing the equality case by certain expression of the second fundamental form. In this paper, first, we present an adapted form for slant submanifolds of a generalized Sasakian space form, similar to the Maslov form, that is always closed. And, in the equality case, we studied under which circumstances the given closed form is also conformal.
\end{abstract}

Keywords: slant submanifolds; generalized Sasakian space forms; closed form; conformal form; Maslov form

\section{Introduction}

It was proven by V. Borrelli, B.-Y. Chen and J. M. Morvan [1], and independently by A. Ros and F. Urbano [2], that if $M$ is a Lagrangian submanifold, with $\operatorname{dim}(M)=m$, of $\mathbb{C}^{m}$, with mean curvature vector $H$ and scalar curvature $\tau$, then $\|H\|^{2} \geq \frac{2(m+2)}{m^{2}(m-1)} \tau$, with equality if and only if $M$ is either totally geodesic or a (piece of a) Whitney sphere. Moreover, they proved that $M$ satisfies the equality case at every point if and only if its second fundamental form $\sigma$ is given by

$$
\sigma(X, Y)=\frac{m}{m+2}\{g(X, Y) H+g(J X, H) J Y+g(J Y, H) J X\},
$$

for any tangent vector fields $X$ and $Y$. Thus, they found a simple relationship between one of the main intrinsic invariants, $\tau$, and the main extrinsic invariant $H$.

It was also proven in [2], that the Maslov form, which is a closed form for a Lagrangian submanifold of $\mathbb{C}^{m}$, is a conformal form if and only if $M$ satisfies (1).

Later, D. E. Blair and A. Carriazo [3] established an analogue inequality for anti-invariant submanifolds in $\mathbb{R}^{2 m+1}$ with its standard Sasakian structure and characterized the equality case with a specific expression of the second fundamental form, similar to Equation (1). In a previous paper [4], we studied the corresponding inequality for slant submanifolds of generalized Sasakian space forms; 
we also characterized the equality case with an specific expression of the second fundamental form; and finally, we presented some examples satisfying the equality case.

Both B.-Y. Chen, [5] and A. Carriazo, [6], have studied the existence of closed forms for slant submanifolds in different environments. The existence of closed forms is particularly interesting, as they provide conditions about submanifolds admitting an immersion in a certain environment.

The purpose of this paper was to obtain some results similar to those of [2] for slant submanifolds of a generalized Sasakian space form. After a section with the main preliminaries, we show that for a slant submanifold of a generalized Sasakian manifold, the Maslov form is not always closed. Therefore, in the following section, we present a form that is always closed for a slant submanifold, so it really plays the role of the Maslov form in the cited papers. Later, if the submanifold satisfies the equality case in the corresponding inequality, that is, if the second fundamental form takes a particular expression [4], we study if the vector field associated with the given form is a conformal vector field.

\section{Preliminaries}

Given a Riemannian manifold $(\widetilde{M}, g)$, a tangent vector field $X$ on $\widetilde{M}$ is called closed if its dual 1 -form is closed. That is equivalent to

$$
g\left(Y, \widetilde{\nabla}_{Z} X\right)=g\left(Z, \widetilde{\nabla}_{Y} X\right)
$$

for all $Y$ and $Z$ on $\widetilde{M}$, where $\widetilde{\nabla}$ is the Levi-Civita connection.

Moreover, $X$ is called conformal if $L_{X} g=\rho g$, for $\rho$ a function on $\widetilde{M}$, where $L$ is the Lie derivative. A closed vector field $X$ is conformal in and only if

$$
\widetilde{\nabla}_{Y} X=f Y,
$$

for any tangent vector field $Y$ on $\widetilde{M}$ and for certain function $f$ on $\widetilde{M}$.

In such a case, considering an orthonormal basis $\left\{e_{1}, \ldots, e_{m}\right\}$ on $\widetilde{M}$, it holds that $\widetilde{\nabla}_{e_{i}} X=f e_{i}$, for $i=1, \ldots, m$.

Now, we will recall some notions about almost-contact Riemannian geometry. For more details about this subject, we recommend the book [7].

An odd-dimensional Riemannian manifold $(\widetilde{M}, g)$ is said to be an almost contact metric manifold if there exists on $\widetilde{M}$, a $(1,1)$ tensor field $\phi$, a unit vector field $\xi$ (called the structure or Reeb vector field) and a 1-form $\eta$, such that

$$
\eta(\xi)=1, \quad \phi^{2}(X)=-X+\eta(X) \xi
$$

and

$$
g(\phi X, \phi Y)=g(X, Y)-\eta(X) \eta(Y)
$$

for any vector fields $X$ and $Y$ on $\widetilde{M}$. In particular, in an almost contact metric manifold we also have

$$
\phi \xi=0, \quad \eta \circ \phi=0 \quad \text { and } \quad \eta(X)=g(X, \xi) .
$$

Such a manifold is said to be a contact metric manifold if $d \eta=\Phi$, where $\Phi(X, Y)=g(X, \phi Y)$ is called the fundamental 2-form of $\widetilde{M}$. The almost contact metric structure of $M$ is said to be normal if $[\phi, \phi](X, Y)=-2 d \eta(X, Y) \xi$, for any $X$ and $Y$. A normal contact metric manifold is called a Sasakian manifold. It can be proven that an almost contact metric manifold is Sasakian if an only if

$$
\left(\widetilde{\nabla}_{X} \phi\right) Y=g(X, Y) \xi-\eta(Y) X,
$$

for any $X$ and $Y$ on $M$. 
In [8], J.A. Oubiña introduced the notion of a trans-Sasakian manifold. An almost contact metric manifold $\widetilde{M}$ is a trans-Sasakian manifold if there exists two functions $\alpha$ and $\beta$ on $\tilde{M}$ such that

$$
\left(\widetilde{\nabla}_{X} \phi\right) Y=\alpha(g(X, Y) \xi-\eta(Y) X)+\beta(g(\phi X, Y) \xi-\eta(Y) \phi X),
$$

for any $X$ and $Y$ on $\tilde{M}$. If $\beta=0, \tilde{M}$ is said to be an $\alpha$-Sasakian manifold. Sasakian manifolds appear as examples of $\alpha$-Sasakian manifolds, with $\alpha=1$. If $\alpha=0, \widetilde{M}$ is said to be a $\beta$-Kenmotsu manifold. Kenmotsu manifolds are particular examples with $\beta=1$. If both $\alpha$ and $\beta$ vanish, then $\widetilde{M}$ is a cosymplectic manifold. In particular, from (4) it is easy to see that the following equation holds for a trans-Sasakian manifold:

$$
\widetilde{\nabla}_{X} \xi=-\alpha \phi X+\beta(X-\eta(X) \xi) .
$$

It was proven by J.C. Marrero that, for dimensions greater or equal than 5 , the only existing trans-Sasakian manifolds are $\alpha$-Sasakian and $\beta$-Kenmotsu ones [9].

In [10], P. Alegre, D.E. Blair and A. Carriazo introduced the notion of a generalized Sasakian space form as an almost contact metric manifold $(\widetilde{M}, \phi, \xi, \eta, g)$ whose curvature tensor is given by

$$
\begin{aligned}
\widetilde{R}(X, Y) Z & =f_{1}\{g(Y, Z) X-g(X, Z) Y\} \\
+ & f_{2}\{g(X, \phi Z) \phi Y-g(Y, \phi Z) \phi X+2 g(X, \phi Y) \phi Z\} \\
& +f_{3}\{\eta(X) \eta(Z) Y-\eta(Y) \eta(Z) X+g(X, Z) \eta(Y) \xi-g(Y, Z) \eta(X) \xi\}
\end{aligned}
$$

where $f_{1}, f_{2}$ and $f_{3}$ are differential functions on $\tilde{M}$. These manifolds are denoted by $\widetilde{M}\left(f_{1}, f_{2}, f_{3}\right)$; generalize the notion of Sasakian space form, $\widetilde{M}(c)$, whose curvature tensor satisfies the expression (6), with

$$
f_{1}=\frac{c+3}{4}, f_{2}=f_{3}=\frac{c-1}{4},
$$

where $c$ is the constant $\phi$-sectional curvature.

Now we recall some general definitions and facts about submanifolds. Let $M$ be a submanifold isometrically immersed in a Riemannian manifold $(\tilde{M}, \mathrm{~g})$. We denote by $\nabla$ the induced Levi-Civita connection on $M$. Thus, the Gauss and Weingarten formulas are respectively given by

$$
\begin{aligned}
& \widetilde{\nabla}_{X} Y=\nabla_{X} Y+\sigma(X, Y), \\
& \widetilde{\nabla}_{X} V=-A_{V} X+D_{X} V,
\end{aligned}
$$

for vector fields $X$ and $Y$ tangent to $M$ and a vector field $V$ normal to $M$, where $\sigma$ denotes the second fundamental form, $A_{V}$ the shape operator in the direction of $V$ and $D$ the normal connection. The second fundamental form and the shape operator are related by

$$
g\left(A_{V} X, Y\right)=g(\sigma(X, Y), V)
$$

$M$ is called a totally geodesic submanifold if $\sigma$ vanishes identically.

We denote by $R$ and $\widetilde{R}$, the curvature tensors of $M$ and $\widetilde{M}$, respectively. They are related by Gauss and Codazzi's equations

$$
\begin{aligned}
& \widetilde{R}(X, Y ; Z, W)= R(X, Y ; Z, W) \\
&+g(\sigma(X, Z), \sigma(Y, W))-g(\sigma(X, W), \sigma(Y, Z)), \\
&(\widetilde{R}(X, Y) Z)^{\perp}=\left(\widetilde{\nabla}_{X} \sigma\right)(Y, Z)-\left(\widetilde{\nabla}_{Y} \sigma\right)(X, Z),
\end{aligned}
$$

where $(\widetilde{R}(X, Y) Z)^{\perp}$ denotes the normal component of $\widetilde{R}(X, Y) Z$ and

$$
\left(\widetilde{\nabla}_{X} \sigma\right)(Y, Z)=D_{X}(\sigma(Y, Z))-\sigma\left(\nabla_{X} Y, Z\right)-\sigma\left(Y, \nabla_{X} Z\right)
$$


is the derivative of Van der Waerden-Bortolotti.

On the other hand, the mean curvature vector $H$ is defined by

$$
H=(1 / \operatorname{dim} M) \operatorname{trace} \sigma,
$$

and $M$ is said to be minimal if $H$ vanishes identically.

The scalar curvature $\tau$ of $M$ at $p \in M$ is defined by

$$
\tau=\sum_{1 \leq i<j \leq \operatorname{dim} M} K\left(e_{i}, e_{j}\right),
$$

where $K\left(e_{i}, e_{j}\right)$ denotes the sectional curvature of $M$ associated with the plane section spanned by $e_{i}$ and $e_{j}$, for any tangent vector fields $e_{i}$ and $e_{j}$ in a local orthonormal frame of $M$.

For a submanifold of an almost contact manifold, we denote

$$
\phi X=T X+N X \text { and } \phi V=t V+n V
$$

the tangent and normal part of $\phi X$ and $\phi V$ for any $X$ tangent vector field and $V$ normal vector field. If the ambient space is trans-Sasakian, taking the tangent and normal part at (4) we obtain:

$$
\begin{gathered}
\left(\nabla_{X} T\right) Y-t \sigma(X, Y)-A_{N Y} X=\alpha(g(X, Y) \xi-\eta(Y) X) \\
+\beta(g(T X, Y) \xi-\eta(Y) T X), \\
\left(\nabla_{X} N\right) Y+\sigma(X, T Y)-n \sigma(X, Y)=-\beta \eta(Y) N X \\
\left(\nabla_{X} t\right) V-A_{n V} X+T A_{V} X=\beta g(N X, V) \xi \\
\left(\nabla_{X} n\right) V+\sigma(X, t V)+N A_{V} X=0 .
\end{gathered}
$$

And from (5):

$$
\begin{gathered}
\nabla_{X} \xi=-\alpha T X+\beta(X-\eta(X) \xi), \\
\sigma(X, \xi)=-\alpha N X .
\end{gathered}
$$

Now, we recall the definition of slant submanifolds. These submanifolds were defined by B.-Y. Chen in [5] on almost Hermitian geometry. Later, A. Lotta defined slant submanifolds on the almost contact metric setting in [11]: given a submanifold $M$ tangent to $\xi$, for each nonzero vector $X$ tangent to $M$ at $p$, such that $X$ is not proportional to $\xi_{p}$, we denote by $\theta(X)$ as the angle between $\phi X$ and $T_{p} M$. Then, $M$ is said to be slant if the angle $\theta(X)$ is a constant, which is independent of the choice of $p \in M$ and $X \in T_{p} M-\left\langle\xi_{p}>\right.$. The angle $\theta$ of a slant immersion is called the slant angle of the immersion. Invariant and anti-invariant immersions are slant immersions with slant angles $\theta=0$ and $\theta=\pi / 2$, respectively. A slant immersion, which is neither invariant nor anti-invariant, is called a proper slant immersion. Slant submanifolds of Sasakian manifolds were studied by J.L. Cabrerizo, A. Carriazo, L.M. Fernández and M. Fernández in [12,13].

From now on, we denote by $m+1=2 n+1$ the dimension of $M$ and $2 m+1=4 n+1$ the dimension of $\widetilde{M}$. We assume $m \geq 2$. Then, for a slant submanifold holds:

$$
\begin{gathered}
T^{2} X=\cos ^{2} \theta(-X+\eta(X) \xi) \\
t N X=\sin ^{2} \theta(-X+\eta(X) \xi) \\
N T X+n N X=0
\end{gathered}
$$

and because of the dimensions,

$$
n^{2} V=-\cos ^{2} \theta V, \quad N t V=-\sin ^{2} \theta V \quad \text { and } \quad T t V+\operatorname{tn} V=0,
$$


for any $X, Y$ tangent vector fields and $V$ normal vector field.

Given a proper slant submanifold $M^{2 n+1}$, with slant angle $\theta$, immersed in an almost contact manifold $\widetilde{M}^{4 n+1}$, we considered an adapted slant reference, [6]; it was built as follows. Given $e_{1}$ a unit tangent vector field, orthogonal to $\xi$, we took:

$$
e_{2}=(\sec \theta) T e_{1}, \quad e_{1 *}=(\csc \theta) N e_{1}, \quad e_{2 *}=(\csc \theta) N e_{2} .
$$

For $k>1$, then proceeding by induction, for each $l=1, \ldots, n-1$, we chose a unit tangent vector field $e_{2 l+1}$ of $M$, such as $e_{2 l+1}$, which is orthogonal to $e_{1}, e_{2}, \ldots, e_{2 l-1}, e_{2 l}, \xi$ and took:

$$
e_{2 l+2}=(\sec \theta) T e_{2 l+1}, \quad e_{(2 l+1) *}=(\csc \theta) N e_{2 l+1}, \quad e_{(2 l+2) *}=(\csc \theta) N e_{2 l+2} .
$$

In this way

$$
\left\{e_{1}, \ldots, e_{m}, \xi, e_{1 *}, \ldots, e_{m *}\right\}
$$

is an orthonormal reference such that $e_{1}, \ldots, e_{m}$ belong to the contact distribution, $\mathcal{D}$ and $e_{1 *}, \ldots, e_{m *}$ are normal to $M$. Moreover, it can be directly computed that:

$$
\begin{array}{lll}
T e_{2 j-1}=(\cos \theta) e_{2 j}, & T e_{2 j}=-(\cos \theta) e_{2 j-1}, & j=1, \ldots, k ; \\
N e_{i}=(\sin \theta) e_{i * \prime} & t e_{i *}=-(\sin \theta) e_{i} & i=1, \ldots, m ; \\
n e_{(2 j-1) *}=-(\cos \theta) e_{(2 j) * \prime} & n e_{(2 j) *}=(\cos \theta) e_{(2 j-1) * \prime} & j=1, \ldots, k .
\end{array}
$$

Finally, a slant submanifold of an $(\alpha, \beta)$ trans-Sasakian generalized Sasakian space form $\widetilde{M}^{2 m+1}\left(f_{1}, f_{2}, f_{3}\right)$, is called $*$-slant submanifold, [4], if its second fundamental form $\sigma$ is given by the following expression:

$$
\begin{aligned}
\sigma(X, Y)= & \frac{m+1}{m+2}\{(g(X, Y)-\eta(X) \eta(Y)) H \\
& +\left(\frac{1}{\sin ^{2} \theta} g(\phi X, H)-\alpha \frac{m+2}{m+1} \eta(X)\right) N Y \\
& \left.+\left(\frac{1}{\sin ^{2} \theta} g(\phi Y, H)-\alpha \frac{m+2}{m+1} \eta(Y)\right) N X\right\}
\end{aligned}
$$

They are specially interesting because it was proven in [4] that this expression of the second fundamental form characterizes the equality case of the following inequality involving the squared mean curvature $\|H\|^{2}$ and the scalar curvature $\tau$ :

$$
(m+1)^{2}\|H\|^{2}-2 \frac{m+2}{m-1} \tau \geq-\frac{m(m+2)}{m-1}\left((m+1) f_{1}+3 f_{2} \cos ^{2} \theta-2 f_{3}-2 \alpha \sin ^{2} \theta\right) .
$$

\section{The Maslov Form}

For any submanifold of any almost contact manifold, we consider the Maslov form $\omega_{H}$ as the dual form of $\phi H$; that is

$$
\omega_{H}(X)=g(X, \phi H),
$$

for any $X$ tangent vector field in the submanifold. We can also define a canonical 1-form on $M$ by

$$
\Theta=\sum_{1=1}^{m} \omega_{i}^{i^{*}}
$$

where $\omega_{i}^{i^{*}}$ are the connection forms given by Cartan's structure equations. 
We can relate these two forms for certain slant submanifolds. In [12], proper slant submanifolds such as for any tangent vector fields $X$ and $Y$ were studied with:

$$
\left(\nabla_{X} T\right) Y=\cos ^{2} \theta(g(X, Y) \xi-\eta(Y) X) .
$$

They were called slant Sasakian submanifolds in [6]; however, we can point that they are $\alpha$-Sasakian manifolds with the induced structure $\bar{\phi}=\sec \theta T$. That aims us to defined slant trans-Sasakian submanifolds as those verifying:

$$
\left(\nabla_{X} \bar{\phi}\right) Y=\bar{\alpha}(g(X, Y) \xi-\eta(Y) X)+\bar{\beta}(g(\bar{\phi} X, Y) \xi-\eta(Y) \bar{\phi} X) .
$$

For a slant trans-Sasakian submanifold of a trans-Sasakian manifold the relation between the structure functions is given by

$$
\sec \theta \bar{\alpha}=\alpha \text { and } \bar{\beta}=\beta .
$$

From (25) and (12) it is deduced that

$$
A_{N Y} X=A_{N X} Y+\alpha \sin ^{2} \theta(\eta(Y) X-\eta(X) Y),
$$

for any $X, Y$ tangent vector fields.

Then, for such a submanifold, the relation between $\Theta$ and the Maslov form is given in the following theorem.

Theorem 1. Let $M^{m+1}$ be a slant trans-Sasakian submanifold of a generalized Sasakian space form $\widetilde{M}^{2 m+1}\left(f_{1}, f_{2}, f_{3}\right)$ endowed with an $(\alpha, \beta)$ trans-Sasakian structure. Then:

$$
\omega_{H}=-\frac{\sin \theta}{m+1}(\Theta+m \alpha \sin \theta \eta)
$$

Proof. Considering an adapted slant basis, it holds

$$
\omega_{H}\left(e_{i}\right)=g\left(e_{i}, \phi H\right)=-g\left(N e_{i}, H\right)=-\sin \theta g\left(e_{i^{*}}, H\right),
$$

for $i=1, \ldots, m$. Moreover,

$$
\Theta=\sum_{l=1}^{2 n} \sum_{i=1}^{2 n} \sigma_{l i}^{l^{*}} \omega^{i}+\sum_{l=1}^{2 n} \sigma_{l \zeta}^{l^{*}} \eta
$$

But,

$$
\sigma_{l \xi}^{l^{*}}=g\left(\sigma\left(e_{l}, \xi\right), e_{l^{*}}\right)=-\csc \theta g\left(N e_{l}, N e_{l}\right)=-\sin \theta
$$

and

$$
\begin{aligned}
\sigma_{l i}^{l^{*}} & =g\left(\sigma\left(e_{l}, e_{i}\right), e_{l^{*}}\right)=\csc \theta g\left(\sigma\left(e_{l}, e_{i}\right), N e_{l}\right) \\
& =\csc \theta g\left(A_{N e_{l}} e_{i}, e_{l}\right)=\csc \theta g\left(A_{N e_{i}} e_{l}, e_{l}\right) \\
& =g\left(\sigma\left(e_{l}, e_{l}\right), e_{i^{*}}\right)=\sigma_{l l}^{i^{*}},
\end{aligned}
$$

where we have used (27); that is, $M$ is a slant trans-Sasakian submanifold.

And therefore, from (30)-(32),

$$
\Theta+m \alpha \sin \theta \eta=\sum_{i=1} 2 n\left(t r \sigma^{i^{*}}\right) \omega^{i} .
$$


As $\sigma(\xi, \xi)=0$ :

$$
H=\frac{1}{m+1} \sum_{j=1}^{m} \sigma\left(e_{j}, e_{j}\right)
$$

Now, from (29) and (33), it holds that

$$
\omega_{H}\left(e_{i}\right)=-\frac{\sin \theta}{m+1} \sum_{j=1}^{2 k} \sigma_{j j}^{i^{*}}=-\frac{\sin \theta}{m+1}(\Theta+m \alpha \sin \theta \eta)\left(e_{i}\right),
$$

for $i=1, \ldots, m$. Finally, as $\omega_{H}(\xi)=g(t H, \xi)=0$, the proof is finished.

Following the same steps that [5] did for slant submanifolds of an almost Hermitian manifold or [6] for an almost contact manifold, and after a long computation, the differentials of $\theta$ and $\eta$ can be proven. The proof is straightforward so we have omitted it.

Lemma 1. Let $M^{m+1}$, a proper slant submanifold of a generalized Sasakian space, form $\widetilde{M}^{2 m+1}$ endowed with an $(\alpha, \beta)$ trans-Sasakian structure, with $M$ tangent to $\xi$ and $m \geq 2$. Then, the 1 -forms $\Theta$ and $\eta$ satisfy:

$$
\begin{aligned}
\mathrm{d} \Theta= & -2 \sin \theta \cos \theta\left(\alpha^{2}+f_{2}(m+1)\right)\left(\sum_{j=1}^{k} \omega^{2 j-1} \wedge \omega^{2 j}-\sum_{j=1}^{k} \omega^{(2 j-1)^{*}} \wedge \omega^{(2 j)^{*}}\right) \\
+\left(-2 \sin ^{2} \theta\left(\alpha^{2}+f_{2}(m+1)\right)+\alpha^{2}+f_{2}-f_{1}-\beta^{2}\right) & \left(\sum_{j=1}^{k} \omega^{2 j-1} \wedge \omega^{(2 j-1)^{*}}+\sum_{j=1}^{k} \omega^{2 j} \wedge \omega^{(2 j)^{*}}\right),
\end{aligned}
$$

and

$$
\begin{aligned}
\mathrm{d} \eta= & -2 \alpha \cos \theta \sum_{j=1}^{k} \omega^{2 j-1} \wedge \omega^{2 j}-2 \alpha \sin \theta \sum_{j=1}^{k} \omega^{2 j-1} \wedge \omega^{(2 j-1)^{*}}- \\
& -2 \alpha \sin \theta \sum_{j=1}^{k} \omega^{2 j} \wedge \omega^{(2 j)^{*}}+2 \alpha \cos \theta \sum_{j=1}^{k} \omega^{(2 j-1)^{*}} \wedge \omega^{(2 j)^{*}},
\end{aligned}
$$

where $\theta$ is the slant angle of $M$.

As we are considering a trans-Sasakian manifold with a dimension greater or equal than 5 , from [9], it must be an $\alpha$-Sasakian or a $\beta$-Kenmotsu manifold. So we distinguish both two cases in the following theorems.

Theorem 2. Let $M^{m+1}$ be a proper slant trans-Sasakian submanifold of a connected generalized Sasakian space form $\widetilde{M}^{2 m+1}\left(f_{1}, f_{2}, f_{3}\right)$ endowed with an $\alpha$-Sasakian structure. Then, the Maslov form is closed if and only if $f_{1}=0$. In such a case, it holds $f_{2}=f_{3}=-\alpha^{2}$.

Proof. As $\widetilde{M}^{2 m+1}$ is $\alpha$-Sasakian, from Proposition 4.1 of [14], $\alpha$ is constant. From (28),

$$
d \omega_{H}=-\frac{\sin \theta}{m+1}(d \Theta+m \alpha \sin \theta d \eta)
$$

Then, from (34) and (35), it is deduced that $\mathrm{d} \omega_{H}=0$ if and only if it holds $\alpha^{2}+f_{2}=0$ and $f_{1}=0$. Moreover, Theorem 4.2 of [14] establishes that both conditions are equivalent, as $f_{1}-\alpha^{2}=f_{2}=f_{3}$.

Remark 1. If the ambient space is a Sasakian space form $\widetilde{M}^{2 m+1}(c)$, the Maslov form is closed if and only if $c=-3$, as it was proved in [6]. 
Theorem 3. Let $M^{m+1}$ be a proper slant trans-Sasakian submanifold of a generalized Sasakian space form $\tilde{M}^{2 m+1}\left(f_{1}, f_{2}, f_{3}\right)$ endowed with a $\beta$-Kenmotsu structure. Then, the Maslov form is closed if and only if

$$
f_{1}=-\beta^{2} \quad \text { and } \quad f_{2}=0
$$

In such a case, it holds $f_{3}=\xi(\beta)$.

Proof. Again from (28), (34) and (35), $\mathrm{d} \omega_{H}=0$ if and only if $f_{2}=0$ and $f_{1}+\beta^{2}=0$. The last condition is obtained from Proposition 4.3 in [14], where it was proven that $f_{1}-f_{3}+\xi(\beta)+\beta^{2}=0$.

Remark 2. We note that on the opposite that for Lagrangian submanifold of $\mathbb{C}^{n}$, [2], or totally real submanifolds of $\mathbb{R}^{2 m+1}$, [3], the Maslov is not always closed. That aims us to look for an adapted form that is closed in more cases.

\section{An Adapted Closed Form}

As the Maslov form is not always closed for slant submanifolds it is necessary to find a new form related with this Maslov form but including the special slant character of the submanifold.

Both the Maslov form and $\Theta$ can be considered forms at $\widetilde{M}$ or $M$. As both $\eta$ and $\Theta$ vanish at $T M^{\perp}$, it is the same defining them on $\widetilde{M}$ or $M$; however, it is not the same considering $d \eta$ or $\left.d \eta\right\rceil_{M}$ and $d \Theta$ or $d \Theta\rceil_{M}$. Although both B.-Y. Chen and A. Carriazo, [5] and [6], studied conditions for $d \omega_{H}$ and $d \Theta$ vanishing at the manifold; their real interest was finding a closed form at the submanifold, not at the manifold.

Therefore, we consider the restrictions of $\Theta$ and $\eta$ at the submanifold. From (34) and (35) it is deduced:

$$
d \eta\rceil_{M}=-2 \alpha \cos \theta \sum_{j=1}^{m} \omega^{2 j-1} \wedge \omega^{2 j}
$$

and

$$
d \Theta\rceil_{M}=-2 \sin \theta \cos \theta\left(\alpha^{2}+f_{2}(m+1)\right) \sum_{j=1}^{m} \omega^{2 j-1} \wedge \omega^{2 j}
$$

So we find that, for obtaining a closed form, the relation between $\Theta$ and $\eta$ is not the given by the Maslov form at (28).

Again, we particularize to $\alpha$-Sasakian or a $\beta$-Kenmotsu manifolds. Firstly, we consider an $\alpha$-Sasakian manifold. It was proven in [14], that if $\alpha \neq 0$ and $\tilde{M}\left(f_{1}, f_{2}, f_{3}\right)$ is connected, then $\alpha$ is constant, and the functions are constant and related by $f_{1}-\alpha^{2}=f_{2}=f_{3}$. We can write:

$$
f_{1}=\frac{c+3 \alpha^{2}}{4}, \quad f_{2}=f_{3}=\frac{c-\alpha^{2}}{4}
$$

From now on, we suppose $\tilde{M}$ is connected.

Lemma 2. Let $M^{m+1}$ be a slant submanifold of an $\alpha$-Sasakian generalized Sasakian space form $\tilde{M}^{2 m+1}\left(f_{1}, f_{2}, f_{3}\right)$, with $\alpha \neq 0$. Then, the form $\Theta-\sin \theta \frac{\alpha^{2}+f_{2}(m+1)}{\alpha} \eta$ is closed at $M$.

Proof. It is directly deduced from (36) and (37) that $\alpha d \Theta-\sin \theta\left(\alpha^{2}+f_{2}(m+1)\right) d \eta=0$, and as $\alpha$ is constant, the result is proven.

Moreover, the field associated to the closed form is

$$
-\frac{m+1}{\sin \theta} t H-\sin \theta\left(m+\frac{\alpha^{2}+f_{2}(m+1)}{\alpha}\right) \xi,
$$


so we already have the following theorem.

Theorem 4. Let $M^{m+1}$ be a slant submanifold of an $\alpha$-Sasakian generalized Sasakian space form $\tilde{M}^{2 m+1}\left(f_{1}, f_{2}, f_{3}\right)$, with $\alpha \neq 0$. Then, the field $t H+\frac{\sin ^{2} \theta}{m+1} \frac{m \alpha+\alpha^{2}+f_{2}(m+1)}{\alpha} \xi$ is closed.

Corollary 1. Let $M^{m+1}$ a slant submanifold of a Sasakian space form $\tilde{M}^{2 m+1}(c)$; the field $t H+\sin ^{2} \theta \frac{c+3}{4} \xi$ is closed.

Note that this result improves the one obtained by A. Carriazo in [6] giving a closed form for a slant submanifold of any Sasakian space form.

Corollary 2. Let $M^{2 m+1}$ be a compact and simply connected manifold. Then, $M$ can not be immersed in a generalized Sasakian space form, $\widetilde{M}^{4 m+1}\left(0,-\alpha^{2},-\alpha^{2}\right)$, endowed with an $\alpha$-Sasakian structure, $\alpha \neq 0$, like a slant submanifold.

Proof. If $M^{m+1}$ is a slant submanifold of $\tilde{M}^{4 m+1}\left(0,-\alpha^{2},-\alpha^{2}\right)$, with an $\alpha$-Sasakian structure. By Theorem 4 the vector field

$$
t H+\frac{\sin ^{2} \theta}{m+1} \frac{m \alpha+\alpha^{2}+f_{2}(m+1)}{\alpha} \xi \neq 0,
$$

is closed, and the corresponding form is also closed. Therefore it represents a cohomology class in $H^{1}(M ; \mathbb{R})$. But, as $M$ is compact, it can not be an exact form. So $H^{1}(M ; \mathbb{R})$ is a nontrivial cohomology class and $M$ could not be simply connected what is a contradiction.

On the other hand, for a $\beta$-Kenmotsu manifold $d \eta=0$ and from Theorem $1, \omega_{H}=-\frac{\sin \theta}{m+1} \Theta$. The following lemma studies when it is a closed form.

Lemma 3. Let $M^{m+1}$ be a proper slant submanifold of a $\beta$-Kenmotsu generalized Sasakian space form $\widetilde{M}^{2 m+1}$, with $M$ tangent to $\xi$ and $m \geq 2$. Then, the Maslov form at $M$ is closed if and only if $f_{2}=0$.

Proof. For a $\beta$-Kenmotsu manifold $\omega_{H}=-\frac{\sin \theta}{m+1} \Theta$. And writing (37) for $\alpha=0$,

$$
d \Theta\rceil_{M}=-2 \sin \theta \cos \theta f_{2}(m+1) \sum_{j=1}^{m} \omega^{2 j-1} \wedge \omega^{2 j} .
$$

Therefore, the Maslov form is closed in $M$ if and only $f_{2}=0$.

Note, that in such a case $f_{1}-f_{3}+\xi(\beta)+\beta^{2}=0$ ([14], Proposition 4.3). Moreover, we observe that, on the opposite that for $\alpha$-Sasakian manifolds, we cannot find a closed form for a slant submanifold of any generalized Sasakian space form with a $\beta$-Kenmotsu structure.

However, for $f_{2}=0$, we have obtained a closed vector field as follows.

Theorem 5. Let $M^{m+1}$ be a slant submanifold of an $\beta$-Kenmotsu generalized Sasakian space form $\tilde{M}^{2 m+1}\left(f_{1}, 0, f_{3}\right)$. Then, the field $t H+\sin ^{2} \theta \frac{m}{m+1} \xi$ is closed.

Again, we can present a topological obstruction for slant immersions: 
Corollary 3. Let $M^{2 m+1}$ be a compact and simply connected manifold. Then, $M$ cannot be immersed in a generalized Sasakian space form, $\tilde{M}^{4 m+1}\left(f_{1}, 0, f_{3}\right)$, endowed with an $\beta$-Kenmotsu structure, as a slant submanifold.

From now on, we will write $t H+a \xi$ and $t H+b \xi$, with

$$
a=\frac{\sin ^{2} \theta}{m+1} \frac{m \alpha+\alpha^{2}+f_{2}(m+1)}{\alpha} \quad \text { and } \quad b=\sin ^{2} \theta \frac{m}{m+1},
$$

for the correspondent closed vector fields.

\section{About Conformal Forms for $\alpha$-Sasakian Space Forms}

As we said in the Introduction, for those Lagrangian submanifolds of $\mathbb{C}^{m}$ verifying the equality case, the Maslov form, that is closed, is also conformal. Now we study if the closed form presented in the previous section is conformal for those slant submanifolds verifying the equality case at (23).

We are considering a connected manifold, so $\alpha, f_{1}, f_{2}$ and $f_{3}$ are constant functions.

We want to compute $\nabla_{X}(t H+a \xi)$, for any $X$ tangent vector field. It is a long computation. Firstly, we compute $\nabla N$ for later use. Using the expression of the second fundamental form of a *slant submanifold, (22), and (20) in (13):

$$
\begin{aligned}
\left(\nabla_{X} N\right) Y=\frac{m+1}{m+2} & \{(g(X, Y)-\eta(X) \eta(Y)) n H-g(X, T Y) H \\
+ & 2\left(\frac{1}{\sin ^{2} \theta} g(\phi X, H)-\frac{m+2}{m+1} \eta(X)\right) n N Y \\
& +\left(\frac{1}{\sin ^{2} \theta} g(\phi Y, H)-\frac{m+2}{m+1} \eta(Y)\right) n N X \\
& \left.-\frac{1}{\sin ^{2} \theta} g(\phi T Y, H) N X\right\}
\end{aligned}
$$

Lemma 4. Let $M$ be *slant submanifold of an generalized Sasakian space form $\tilde{M}\left(f_{1}, f_{2}, f_{3}\right)$ endowed with an $\alpha$-Sasakian structure. For every $X$ tangent vector field belonging to the contact distribution it holds:

$$
\begin{aligned}
\nabla_{X}(t H+a \xi)= & -g\left(D_{X} H, N X\right) X+\left(\frac{1}{\sin ^{2} \theta} \frac{m+1}{m+2} g^{2}(H, N X)-a\right) T X \\
& -\frac{3}{\sin ^{2} \theta} \frac{m+1}{m+2} g(H, N X) t n H \\
& +\frac{1}{\sin ^{2} \theta} \frac{m+1}{m+2} g(H, n N X) t H+g(H, n N X) \xi .
\end{aligned}
$$

Proof. Firstly, from Codazzi's equation we will compute $D_{X} H$, and after, $\nabla_{X}(t H+a \xi)$. Writing Codazzi's equation, (9), for a generalized Sasakian space form, for any unit orthogonal $X, Y$ tangent vector fields in the contact distribution, using (3) (6), $R(X, Y) Y)^{\perp}$ gives:

$$
\begin{aligned}
& 3 \frac{m+2}{m+1} f_{2} g(X, T Y) N Y=D_{X} H+3 \frac{m+2}{m+1} g(Y, T X) N Y \\
& +\frac{2}{\sin ^{2} \theta}\left\{g\left(\left(\nabla_{X} N\right) Y, H\right) N Y+g\left(N Y, D_{X} H\right) N Y+g(N Y, H)\left(\nabla_{X} N\right) Y\right\} \\
& +\frac{1}{\sin ^{2} \theta}\left\{-g\left(\left(\nabla_{Y} N\right) X, H\right) N Y-g\left(\left(\nabla_{Y} N\right) Y, H\right) N X-g(N X, H)\left(\nabla_{Y} N\right) Y\right. \\
& \left.-g\left(N X, D_{Y} H\right) N Y-g\left(N Y, D_{Y} H\right) N X-g(N Y, H)\left(\nabla_{Y} N\right) X\right\}
\end{aligned}
$$


Then, using (40), we obtain:

$$
\begin{aligned}
& 3 \frac{m+2}{m+1}\left(f_{2}+1\right) g(X, T Y) N Y=D_{X} H \\
& +\frac{1}{\sin ^{2} \theta}\left\{2 g\left(N Y, D_{X} H\right) N Y-g\left(N X, D_{Y} H\right) N Y-g\left(N Y, D_{Y} H\right) N X\right\} \\
& +\frac{1}{\sin ^{2} \theta} \frac{m+1}{m+2}\left\{-g(N X, H) n H-\frac{2}{\sin ^{2} \theta} g(N Y, H) g(n N Y, H) N X-3 g(N Y, H) g(X, T Y) H\right. \\
& \left.\left(-3 g(X, T Y)\|H\|^{2}+\frac{4}{\sin ^{2} \theta} g(N X, H) g(n N Y, H)-\frac{2}{\sin ^{2} \theta} g(N Y, H) g(n N X, H)\right) N Y\right\} .
\end{aligned}
$$

At this point, we use that, taking into account Corollary $1, t H+a \xi$ is a closed vector field.

$$
g\left(\nabla_{X}(t H+a \xi), Y\right)=g\left(\nabla_{Y}(t H+a \xi), X\right)
$$

Then, using (16)

$$
g\left(\nabla_{X} t H, Y\right)=g\left(\nabla_{Y} t H, X\right)-2 a g(T Y, X)
$$

and therefore, (14) gives

$$
\begin{aligned}
& g\left(N X, D_{Y} H\right)=-g\left(X, t D_{Y} H\right)=-g\left(X, \nabla_{Y} t H-A_{n H} Y+T A_{H} Y\right) \\
& =g\left(N Y, D_{X} H\right)+2 a g(T X, Y)-g(\sigma(T Y, X), H)+g(\sigma(Y, T X), H) .
\end{aligned}
$$

Now, using (22) carries to

$$
\begin{aligned}
g\left(N X, D_{Y} H\right)= & g\left(N Y, D_{X} H\right)+2 a g(T X, Y)+\frac{m+1}{m+2} 2 g(Y, T X)\|H\|^{2} \\
& +\frac{m+1}{m+2} \frac{2}{\sin ^{2} \theta}(g(N Y, H) g(N T X, H)-g(N X, H) g(N T Y, H)) .
\end{aligned}
$$

So (43) gives

$$
\begin{aligned}
3 \frac{m+2}{m+1}\left(f_{2}+1\right) g(X, T Y) N Y=D_{X} H+\frac{1}{\sin ^{2} \theta}\left\{g\left(N Y, D_{X} H\right) N Y-g\left(N Y, D_{Y} H\right) N X\right\} \\
+\frac{1}{\sin ^{2} \theta} \frac{m+1}{m+2}\left\{\left(-2 a \frac{m+2}{m+1} g(T X, Y)+g(Y, T X)\|H\|^{2}+\frac{2}{\sin ^{2} \theta} g(N X, H) g(n N Y, H)\right) N Y\right. \\
\left.-g(N X, H) n H-\frac{2}{\sin ^{2} \theta} g(N Y, H) g(n N Y, H) N X+3 g(N Y, H) g(Y, T X) H\right\} .
\end{aligned}
$$

Now, for dimensions over or equal than 5, we can consider $X$ orthogonal to $Y$ and $T Y$. Multiplying by $N X$,

$$
\begin{aligned}
0 & =g\left(D_{X} H, N X\right)-g\left(N Y, D_{Y} H\right) \\
& +\frac{1}{\sin ^{2} \theta} \frac{m+1}{m+2}\{-g(N X, H) g(n N X, H)-2 g(N Y, H) g(n N Y, H)\} .
\end{aligned}
$$

Interchanging $X$ and $Y$ at (47), and adding it to the previous equation:

$$
g(N X, H) g(n N X, H)=-g(N Y, H) g(n N Y, H) .
$$

For $T Y$, that is also orthogonal to $X, T X$,

$$
g(N X, H) g(n N X, H)=-g(N T Y, H) g(n N T Y, H)=\cos ^{2} \theta g(N Y, H) g(n N Y, H) .
$$


From (48) and (51), we get $g(N X, H) g(n N X, H)=0$ for every $X$ unit vector field in the contact distribution. Moreover, developing $0=g(N(X+Y), H) g(n N(X+Y), H)$, we obtain

$$
g(N X, H) g(n N Y, H)=-g(N Y, H) g(n N X, H) .
$$

Also, at (47), we get

$$
g\left(D_{X} H, N X\right)=g\left(N Y, D_{Y} H\right),
$$

so $g\left(D_{X} H, N X\right)$ is independent of $X$ unit vector field in the contact distribution.

Now, multiplying (46) by $N Y$,

$$
0=2 g\left(D_{X} H, N Y\right)+\frac{3}{\sin ^{2} \theta} \frac{m+1}{m+2} g(N X, H) g(n N Y, H) .
$$

But (44) for any $X$, a unitary vector field orthogonal to $Y, T Y$, in the contact distribution it states:

$$
g\left(N X, D_{Y} H\right)-g(\sigma(T X, Y), H)=g\left(N Y, D_{X} H\right)-g(\sigma(T Y, X), H) .
$$

Using (52) and (22) at (53)

$$
\frac{-7}{2 \sin ^{2} \theta} \frac{m+1}{m+2} g(N X, H) g(n N Y, H)=\frac{-7}{2 \sin ^{2} \theta} \frac{m+1}{m+2} g(N Y, H) g(n N X, H),
$$

where $X, Y$ can be interchanged. Comparing (50) with (54) it is proven that

$$
g(N X, H) g(n N Y, H)=0,
$$

and consequently, by (52),

$$
g\left(D_{X} H, N Y\right)=0,
$$

for each $X$ orthogonal to $Y$ and $T Y$ at the contact distribution.

It only rests on us to compute $g\left(D_{X} H, N T X\right)$ in order to know $D_{X} H$. Multiplying (46) by NTX we obtain:

$$
g\left(D_{X} H, N T X\right)=-\frac{\cos ^{2} \theta}{\sin ^{2} \theta} \frac{m+1}{m+2} g(H, N X)^{2} .
$$

Therefore, taking an orthogonal basis $\left\{e_{1}^{*}, \ldots, e_{n}^{*}\right\}$ at $T^{\perp} M$,

$$
\begin{aligned}
D_{X} H & =\sum g\left(D_{X} H, e_{j}^{*}\right) e_{j}^{*}= \\
& =\frac{1}{\sin ^{2} \theta} g\left(D_{X} H, N X\right) N X-\frac{1}{\sin ^{4} \theta} \frac{m+1}{m+2} g(H, N X)^{2} N T X,
\end{aligned}
$$

for any $X$ unit tangent field orthogonal to $\xi$.

Finally, for any $X$ at the contact distribution, and any $Z$ tangent vector field,

$$
\begin{gathered}
g\left(\nabla_{X}(t H+a \xi), Z\right)=g\left(\nabla_{X} t H, Z\right)-a g(T X, Z) \\
=g\left(t \nabla_{X} H+A_{n H} X-T A_{H} X, Z\right)-a g(T X, Z) \\
=-g\left(D_{X} H, N Z\right)+g(n H, h(X, Z))+g(H, h(X, T Z))-a g(T X, Z) \\
=-g\left(g\left(D_{X} H, N X\right) N X-\frac{1}{\sin ^{4} \theta} \frac{m+1}{m+2} g(H, N X)^{2} N T X, N Z\right) \\
+\frac{m+1}{m+2}\left(\frac{1}{\sin ^{2} \theta} g(N X, H) g(N Z, n H)+\left(\frac{1}{\sin ^{2} \theta} g(N Z, H)-\frac{m+2}{m+1} \eta(Y)\right) g(N X, n H)\right)
\end{gathered}
$$




$$
\begin{gathered}
+\frac{m+1}{m+2}\left(\frac{1}{\sin ^{2} \theta} g(N X, H) g(N T Z, H)+\frac{1}{\sin ^{2} \theta} g(N T Z, H) g(N X, H)\right) \\
-a g(T X, Z),
\end{gathered}
$$

where we used (22). This last equation, using (19), direct gives the desired expression of $\nabla_{X} t H+$ $\frac{c-1}{4} \cos ^{2} \theta \xi$.

The quid point of the above proof is to deduce, from Codazzi's equation and the expression of the second fundamental form, that $g\left(D_{X} H, N X\right)$ is independent of $X$ and also that $g\left(D_{X} H, N Y\right)=0$ for $Y$ orthogonal to $X, T X$. This is the same sketch than A. Ros and F. Urbano did in [2].

Now, we repeat the same steps in order to obtain $\nabla_{\xi}(t H+a \xi)$.

Lemma 5. Let $M$ be *-slant submanifold of a Sasakian space form $\widetilde{M}(c)$; it holds:

$$
\nabla_{\xi}(t H+a \xi)=-T t H .
$$

Proof. Using that, from Corollary $1, t H+a \xi$ is a closed vector field,

$$
g\left(\nabla_{\xi}(t H+a \xi), Y\right)=g\left(\nabla_{Y}(t H+a \xi), \xi\right),
$$

so using (16),

$$
g\left(\nabla_{\xi} t H, X\right)=g\left(\nabla_{X} t H, \xi\right)=-g\left(t H, \nabla_{X} \xi\right)=g(t H, T X)=-g(T t H, X),
$$

for any $X$ tangent vector field, which finishes the proof.

Theorem 6. Let $M$ be *slant submanifold of a generalized Sasakian space form $\widetilde{M}\left(f_{1}, f_{2}, f_{3}\right)$, endowed with an $\alpha$-Sasakian structure. Then, for every $X$ tangent vector field it holds:

$$
\begin{aligned}
\nabla_{X}(t H+a \xi)= & \left(-g\left(D_{X} H, N X\right)+\eta(X) g(H, n N X)\right)(X-\eta(X) \xi) \\
& +\left(\frac{1}{\sin ^{2} \theta} \frac{m+1}{m+2} g(H, N X)^{2}-a\right) T X \\
& +\left(\frac{-3}{\sin ^{2} \theta} \frac{m+1}{m+2} g(H, N X)+\eta(X)\right) t n H \\
& +\frac{1}{\sin ^{2} \theta} \frac{m+1}{m+2} g(H, n N X) t H+g(H, n N X) \xi .
\end{aligned}
$$

Proof. It is a direct consequence of Lemmas 4 and 5.

So, in general, for a *-slant submanifold of a generalized Sasakian space form, the closed form is not conformal. However, for the corresponding vector field, the covariant derivative with respect to $X$ is in the direction of $X, T X, t n H$ and $\xi$.

\section{About Conformal Forms for $\beta$-Kenmotsu Space Forms}

At Section 4 we obtained that, for a $\beta$-Kenmotsu generalized Sasakian space form $\widetilde{M}\left(f_{1}, 0, f_{3}\right)$, the vector field $t H+\sin ^{2} \theta \frac{m}{m+1} \xi=t H+b \xi$ is always closed. So, the associated form plays the role of the Maslov form for Lagrangian submanifolds of Kaehler manifolds. In this section we study if it is conformal for a *-slant submanifold.

The study is similar to the one made at Section 5 , so we omit the proofs. 
Lemma 6. Let $M$ be ${ }^{*}$-slant submanifold of a $\beta$-Kenmotsu generalized Sasakian space form $\widetilde{M}\left(f_{1}, 0, f_{3}\right)$. Then, for every $X$ tangent vector field belonging to the contact distribution it holds:

$$
\begin{aligned}
\nabla_{X}(t H+b \xi)= & \left(-g\left(D_{X} H, N X\right)+\beta \sin ^{2} \theta \frac{m}{m+1}\right) X \\
& +\frac{m+1}{m+2}\left(\frac{1}{\sin ^{2} \theta} g(H, N X)^{2}-\|H\|^{2}\right) T X \\
& -\frac{3}{\sin ^{2} \theta} \frac{m+1}{m+2} g(H, N X) t n H \\
& +\frac{1}{\sin ^{2} \theta} \frac{m+1}{m+2} g(H, n N X) t H+\beta g(N X, H) \xi .
\end{aligned}
$$

Again, the quid point of the proof is to deduce, from Codazzi's equation and the expression of the second fundamental form, that $g\left(D_{X} H, N X\right)$ is independent of $X$ and that $g\left(D_{X} H, N Y\right)=0$ for $X$ orthogonal to $Y$.

Now, we repeat the same steps in order to obtain $\nabla_{\xi}(t H+b \xi)$.

Lemma 7. Let $M$ be *-slant submanifold of a $\beta$-Kenmotsu space form $\widetilde{M}\left(f_{1}, 0, f_{3}\right)$; it holds:

$$
\nabla_{\xi} t H=-\beta t H .
$$

Finally, we get:

Theorem 7. Let $M$ be ${ }^{*}$-slant submanifold of a $\beta$ Kenmotsu space form $\tilde{M}\left(f_{1}, 0, f_{3}\right)$. Then, for every $X$ tangent vector field it holds:

$$
\begin{aligned}
& \nabla_{X}(t H+b \xi)= \\
& =\left(-g\left(D_{X} H, N X\right)+\beta \eta(X) g(H, n N X)+\beta \sin ^{2} \theta \frac{m}{m+1}\right)(X-\eta(X) \xi) \\
& +\frac{m+1}{m+2}\left(\frac{1}{\sin ^{2} \theta} g(H, N X)^{2}-\|H\|^{2}\right) T X-\frac{3}{\sin ^{2} \theta} \frac{m+1}{m+2} g(H, N X) t n H \\
& +\frac{1}{\sin ^{2} \theta} \frac{m+1}{m+2} g(H, n N X) t H-\beta \eta(X) t H+\beta g(N X, H) \xi .
\end{aligned}
$$

Proof. It is a direct consequence from Lemmas 6 and 7.

Again, for a *-slant submanifold of a $\beta$-Kenmotsu generalized Sasakian space form, the closed form is not conformal. However, for the corresponding vector field, the covariant derivative with respect to $X$ is in the direction of $X, T X, t H, t n H$ and $\xi$.

Author Contributions: All the authors contributed equally to this work.

Conflicts of Interest: The authors declare no conflict of interest.

\section{References}

1. Borrelli, V.; Chen, B.Y.; Morvan, J.M. Une caractérisation géométrique de la sphère de Whitney. C. R. Acad. Sci. Paris 1995, 321, 1485-1490.

2. Ros, A.; Urbano, F. Lagrangian submanifolds of $\mathbf{C}^{n}$ with conformal Maslov form and the Whitney sphere. J. Math. Soc. Jpn. 1998, 50, 203-226. [CrossRef]

3. Blair, D.E.; Carriazo, A. The contact Whitney sphere. Note Mat. 2000, 20, 125-133.

4. Alegre, P. Barrera, J.; Carriazo, A. A new class of slant submanifolds in generalized Sasakian space forms. Appear Med. J. Math. 2020, under review.

5. Chen, B.Y. Geometry of Slant Submanifolds; Katholieke Universiteit Leuven: Leuven, Belgium, 1990. 
6. Carriazo, A. Obstructions to slant immersions in contact manifolds. Ann. Mat. Pura Appl. 2001, 179, $459-470$. [CrossRef]

7. Blair, D.E. Contact Manifolds in Riemannian Geometry, Lecture Notes in Mathematics 509; Springer: New York, NY, USA, 1976.

8. Oubi na, J.A. New classes of almost contact metric structures. Publ. Math. Debrecen 1985, 32, 187-193.

9. Marrero, J.C. The Local Structure of Trans-Sasakian Manifolds. Ann. Mat. ura Appl. CLXII 1992, 162, 77-86. [CrossRef]

10. Alegre, P.; Blair, D.; Carriazo, A. Generalized Sasakian-space-forms. Israel J. Math. 2004, 141, $157-183$. [CrossRef]

11. Lotta, A. Slant submanifolds in contact geometry. Bull. Math. Soc. Roum. 1996, 39, 183-198.

12. Cabrerizo, J.L.; Carriazo, A.; Fernández, L.M.; Fernández, M. Slant submanifolds in Sasakian manifolds. Glasgow Math. J. 2000, 42, 125-138. [CrossRef]

13. Cabrerizo, J.L.; Carriazo, A.; Fernández, L.M.; Fernández, M. Structure on a slant submanifold of a contact manifold. Indian J. Pure Appl. Math. 2000, 31, 857-864.

14. Alegre, P.; Carriazo, A. Structures on generalized Sasakian-space-forms. Differ. Geom. Appl. 2008, 26, 656-666. [CrossRef]

(C) 2019 by the authors. Licensee MDPI, Basel, Switzerland. This article is an open access article distributed under the terms and conditions of the Creative Commons Attribution (CC BY) license (http:/ / creativecommons.org/licenses/by/4.0/). 